\title{
Effects of repeated annual influenza vaccination on vaccine sero-response in young and elderly adults
}

\author{
W.E.P. Beyer*, A.M. Palache*, M.J.W. Sprenger†, E. Hendriksent,
} J.J. Tukker§, R. Darioliף, G.L. van der Water*, N. Masurel* and A.D.M.E. Osterhaus*\|

Three cohort studies in adults were performed during the periode from 1986 to 1989. Eight hundred and eighty-four subjects were, one or more times, immunized with influenza vaccines, and pre-and post-vaccination antibody titres were determined by hemagglutination inhibition tests. One thousand and one hundred and nineteen vaccination events in 681 subjects could be analysed by a comparison, per trial and per influenza (sub) type, between groups with and without influenza vaccination in previous years. Effect size, odds ratio and protection rate difference, were used as effect measures. Subjects with previous vaccination showed higher pre-vaccination antibody than those without. The average change of the post-vaccination proportion of subjects with high antibody titre value to previous vaccination, was $+9.4 \%$ (95\% CI: +5.3 to $13.6 \%)$ for A-H3N2 vaccine components, $-2.1 \%$ (-8.1 to $3.9 \%$, not significant) for $\mathrm{A}-\mathrm{HINI}$ and $-10.6 \%$ (-16.5\% to $-4.8 \%$ ) for $B$. In a linear regression model, pre-vaccination titres and the status of previous vaccination were identified as factors significantly influencing post-vaccination titres. These findings are discussed in the context of a short review of the literature. It is concluded that the status of previous vaccination should always be addressed as an independent factor in serological vaccination studies. Copyright (C) 1996 Elsevier Science Ltd.

Keywords: Influenza vaccine; hemagglutination inhibition test; vaccine sero response; repeated vaccination

Active immunization with inactivated vaccines against influenza virus types $A$ and $B$ in subjects at risk of developing serious complications after influenza infection, has been advised to be repeated annually to comply with antigenic drift of the viruses and decrease of antibody levels with time. This policy, however, has been questioned by field studies in the 1970s, which suggested a decrease of protective immunity upon annually repeated vaccination ${ }^{1-3}$. Although the methods of these studies have been criticized ${ }^{4}$ and more recent studies ${ }^{5-7}$ have not confirmed these findings, the value of annual influenza vaccination still remains a subject of discussion.

*WHO National Influenza Centre, Institute of Virology, Erasmus University Rotterdam, P.O. Box 1738, 3000 DR Rotterdam, The Netherlands. †Department of Infectious Diseases and Epidemiology, National Institute of Public Health and Environmental Protection, Bilthoven, The Netherlands. ¥Nursing Home Den Ooiman, Doetinchem, The Netherlands. §Department of Pharmacy, University of Utrecht, Utrecht, The Netherlands. TDepartment of Internal Medicine, University of Lausanne, Lausanne, Switzerland. |To whom correspondence should be addressed. (Received 7 April 1995; accepted 26 February 1996)
Field vaccination studies are difficult to perform due to the poor predictability of influenza outbreaks, which makes power-calculations troublesome. The most important parameters of efficacy are reduction of mortality and severe morbidity which may not occur frequently thus requiring large numbers of participants in efficacy trials. Moreover, since a beneficial effect of influenza vaccination has been established, it is no longer ethically acceptable to perform double-blind, prospective field studies in groups at risk of developing serious complications. Therefore, serological studies with hemagglutination inhibition (HI) serum antibody titres as a surrogate marker for real vaccine efficacy, are usually performed, with divergent results: sometimes a lower ${ }^{7,8}$, and sometimes a higher ${ }^{9}$ postvaccination serum antibody titre was observed in subjects with a vaccination history when compared to those not vaccinated before, and in other studies, post-vaccination antibody titres were not significantly affected by previous vaccinations ${ }^{5,10-12}$.

Here we present the results of three different cohort studies, performed in primed populations during an interpandemic period (1986-1989) with low activity of naturally occurring influenza. 
Table 1 Vaccines used in three trial cohort studies

\begin{tabular}{|c|c|c|c|c|c|c|c|}
\hline \multirow[b]{2}{*}{ Season } & \multirow[b]{2}{*}{ Composition } & \multicolumn{2}{|l|}{ YA } & \multicolumn{2}{|l|}{$A E$} & \multicolumn{2}{|l|}{$\mathrm{RE}$} \\
\hline & & Type $^{a}$ & Dosage $(\mu \mathrm{g} \mathrm{HA})$ & Type & Dosage $(\overline{\mu \mathrm{g} \mathrm{HA}})$ & Type & Dosage $(\mu \mathrm{g} \mathrm{HA})$ \\
\hline 1986 & $\begin{array}{l}\text { A/Mississippi/1/85 (H3N2) } \\
\text { A/Chile/1/83 } \\
\text { B/Ann Arbor/1/86 } \\
\text { A/Taiwan/1/86 (H1N1) }\end{array}$ & su & $\begin{array}{l}10 \text { or } 20^{b} \\
10 \text { or } 20 \\
10 \text { or } 20\end{array}$ & su & $\begin{array}{l}10 \\
10 \\
15\end{array}$ & WV & $\begin{array}{l}10 \text { or } 20^{c} \\
10 \text { or } 20 \\
15 \text { or } 30 \\
15 \text { or } 30\end{array}$ \\
\hline 1987 & $\begin{array}{l}\text { A/Leningrad/360/86 (H3N2) } \\
\text { A/Taiwan/1/86 (H1N1) } \\
\text { B/Ann Arbor/1/86 }\end{array}$ & su & $\begin{array}{l}10 \\
10 \\
10\end{array}$ & SU & $\begin{array}{l}10 \\
10 \\
10\end{array}$ & WV & $\begin{array}{l}10 \\
10 \\
15\end{array}$ \\
\hline 1988 & $\begin{array}{l}\text { A/Sichuan/2/87 (H3N2) } \\
\text { A/Taiwan/1/86 (H1N1) } \\
\text { B/Beying/1/87 }\end{array}$ & SU & $\begin{array}{l}10 \\
10 \\
10\end{array}$ & SU & $\begin{array}{l}10 \\
10 \\
10\end{array}$ & WV & $\begin{array}{l}10 \\
10 \\
10\end{array}$ \\
\hline 1989 & $\begin{array}{l}\text { A/Shanghai/11/87 (H3N2) } \\
\text { A/Taiwan/1/86 (H1N1) } \\
\text { B/Yamagata/16/87 }\end{array}$ & & & SU & $\begin{array}{l}10 \\
10 \\
10\end{array}$ & WV & $\begin{array}{l}10 \\
10 \\
10\end{array}$ \\
\hline
\end{tabular}

${ }^{a} \mathrm{WV}$, whole virus vaccine; SU, subunit vaccine. ${ }^{\circ} \mathrm{A}$ dose response trial with two dosages. ${ }^{\circ}$ Dosage scheme, including booster vaccinations, described in Ref. 14

\section{MATERIALS AND METHODS}

\section{Vaccimees and trials}

The first two cohort studies (designated YA, young adults, and $\mathrm{AE}$, ambulatory elderly) were part of open vaccination studies to comply with regulatory requirements, as described elsewhere ${ }^{13}$. Young, clinically healthy adults were recruited from medical schools and other teaching institutions in The Netherlands. Ambulatory elderly with $(70 \%)$ or without $(30 \%)$ agerelated chronic diseases, were recruited from a general geriatric outpatients' department in Switzerland. Before intake, previous vaccinations against influenza were documented. In total, 237 young adult and 221 elderly subjects were vaccinated once or more times in the period from 1986 to 1989 , respectively. During the study period, no cases of influenza-like illness (ILI) were reported by the vaccinees.

The third cohort study (designated RE, resident elderly) took place in a Dutch rural nursing home with 275 beds (135 for somatic patients and 140 for psychogeriatric patients). Before 1986, only a few patients received influenza vaccine on their own request. But. when in January/February 1986 a large influenza A-H3N2 epidemic affected 103 of the patients $(37 \%)^{14}$, general vaccinations were performed every year since autumn 1986. The annual vaccination rate in the nursing home varied between 71 and $81 \%$, involving 426 patients who were vaccinated once or more times between 1986 and 1989 . Most of these subjects $(97 \%)$ were older than 60 years of age at the moment of first vaccination. The vaccination history of new entries could not always be traced back in patients with dementia. In March 1989 a small epidemic of ILI occurred in 19 patients (influenza B serologically confirmed in seven patients).

All volunteers (or in case of dementia, their relatives) gave informed consent to participate in the trials. For the cohort studies YA and AE, Medical Ethical Committee approval was obtained.

For this study, vaccinees were selected according to the following post-hoc criteria:

(1) Age: In cohort studies AE and RE only subjects born in 1927 or earlier were included; in cohort study YA only subjects born after 1927 were included.
(2) History of previous vaccinations: Subjects who, at the entry of the cohort studies, recorded previous vaccinations against influenza within the last 5 years, were excluded. Moreover, subjects who were repeatedly vaccinated in the course of a cohort study, were included if the vaccination sequence was not interrupted by a year without vaccination. For example, a subject vaccinated in 1987 and 1989 , but not in 1988, was included in the trial 1987, but not in 1989.

(3) Lack of effect group: The trials of 1986 were not included in the serological analysis as they all consisted of not previously vaccinated subjects (i.e. no comparison according to status of previous vaccinations possible).

(4) Complete serology: Subjects with incomplete either pre- or post-vaccination sera due to lack of compliance or organization mistakes, or subjects with less than 15 days between pre- and post-vaccination serum, or subjects with missing titres for either serum or either vaccine component due to laboratory limitations, were excluded from the actual trial (but not necessarily from the following trials).

\section{Vaccine preparations and doses}

Inactivated influenza vaccines (Influvac whole-virus vaccine, Influvac subunit vaccine; Solvay-Duphar BV, Weesp, The Netherlands) contained strains of influenza A-H3N2, A-H1N1 and B, according to the annual recommendations of the World Health Organization. As shown in Table 1 , the A-H1N1 component remained unchanged for most years while the A-H3N2 and B components were replaced by new variants every 1 or 2 years. The influenza vaccine type was whole-virus for cohort study RE, and subunit for cohort studies YA and AE. Vaccine doses varied between 10 and $15 \mu \mathrm{g}$ HA per strain, except for two dose-response trials in 1986. Vaccines were administered intramuscularly or deep subcutaneously.

\section{Serological methods}

Serum specimens were collected prior to vaccination (pre-vaccination serum) and again after at least 15 days 
(post-vaccination serum). Samples were stored frozen prior to laboratory determinations of homologous hemagglutination-inhibition (HI) antibody titres. All sera of a trial were titrated simultaneously and in duplicate. Cohort studies YA and AE were titrated in the laboratory of Solvay-Duphar, with a test-antigen concentration of 4-8 hemagglutination units (HAU) ${ }^{15}$, without ether-treatment of influenza B test-antigen. With this method, protection is supposed to be associated with a titre of $\geq 40$ (influenza $A$ ) and $\geq 20$ (influenza B). Negative sera (no inhibition in a 1:8 dilution) were arbitrarily recorded as 4 for calculations. Cohort study RE was titrated at the National Influenza Centre, by a similar method, but with a lower test-antigen concentration of $3 \mathrm{HAU}^{16}$, and with ether-treatment of influenza $B$ test-antigen ${ }^{17}$; this method produced high absolute titres and was described as being associated with a protection threshold of $\geq 100$ (influenza $A)^{18}$ and $\geq 200$ (influenza B) ${ }^{19}$. Negative sera (no inhibition in a 1:9 dilution) were arbitrarily recorded as 5 . Titre values were transformed to decadic logarithms.

Association of the "protection thresholds" (40/20 or $100 / 200$ ) with real protection is controversial. For convenience, we called subjects surpassing the appropriate titre thresholds "protected subjects", rather than "subjects with high antibody titre".

\section{Statistical methods}

As titrations were performed separately per trial, no comparisons could be made between, but only within trials. Per trial and per vaccine component, pre- and post-vaccination geometric mean titres (pre-GMT, postGMT), and pre- and post-vaccination proportions of protected subjects (pre-PR, post-PR) were calculated for subgroups with previous vaccination (effect groups) and without previous vaccination (control groups).

Per trial and per vaccine component, the effect of previous vaccination was estimated by measuring the differences between effect and control groups. Three effect measures were calculated:

(1) Effect size (ES) (according to Dawson-Saunders et $a .^{20}$, defined as:

$$
E S=\left(\mathrm{GMT}_{1}-\mathrm{GMT}_{0}\right) / \mathrm{S} . \mathrm{D} \cdot \text { pooled } \cdot
$$

where $\mathrm{GMT}_{0}$ and $\mathrm{GMT}_{1}$ represent the geometric mean titres of the control and effect groups, respectively, and S.D.pooled is the pooled standard deviation.

(2) Logarithm of odds ratio (O.R.), defined as:

$$
\ln O . R .=\ln (A+0.5)^{*}(D+0.5) /\left((B+0.5)^{*}(C+0.5)\right) .
$$

where $A$ and $C$ are the numbers of protected subjects in the control and effect groups, respectively, and $B$ and $D$ are the numbers of not protected subjects in control and effect group, respectively.

(3) Rate difference $(R D)$, defined as:

$R D=P R_{1}-P R_{0}$.

where $P R_{0}$ and $P R_{1}$ are the proportions of protected subjects in the control and effect groups, respectively.
$E S$-, $\ln O . R$ - and $R D$-values are 0 in case of no difference between effect and control group (i.e. no effect of previous vaccination); they are positive in case of higher titres or percentage of protection in the group with previous vaccinations, compared to the control group, and vice versa. The calculation of $95 \%$ confidence intervals allows to define the significance of the effect measures

Per (sub)type, effect measures from all trials were pooled by a one-step technique of meta-analysis as described previously (Yusuf-Peto modified CochranMantel-Haenzel method ${ }^{21}$ ) resulting in pooled values for influenza A-H3N2, A-H1N1 and B, respectively. The validity of the pooling procedure was tested by a $\chi^{2}$-test for homogeneity between trials, according to Breslow and Day 22

Per trial and per vaccine component, post-GMT were subjected to linear regression with status of previous vaccinations (i.e. 0 for control groups, 1 for effect groups) as independent variable. In a first model of linear regression also other factors were included as independent variables: pre-vaccination titre, year of birth, gender, days between vaccination and drawing of post-vaccination blood specimen, number of previous vaccinations (one to three times), underlying diseases (for cohort studies AE and RE), vaccine dosages in 1986 (for cohort studies YA and RE), and clinically or virologically confirmed ILI in January/February 1986 and March 1989 (for cohort study RE). In a consecutive model, only those factors were included which significantly contributed to post-vaccination GMT in the first model. In a similar procedure, post-vaccination percentages of protection were subjected to logistic regression.

For all statistical procedures, a $P$-value of 0.05 or less was considered to indicate statistical significance. Calculations were done on a personal computer using a statistical software package (SPSS/PC+4.0; Microsoft Corporation, Redmond/Washington, USA).

\section{RESULTS}

\section{Final numbers of vaccinees with complete serology}

As shown in Table 2, in three cohort studies 1715 vaccinations were performed, involving 884 subjects who were vaccinated once or more times during the period from 1986 to 1989. After applying the post-hoc selection criteria described in Materials and Methods, 1119 paired sera of 681 subjects entered the analysis: in cohort study YA, 181 subjects with a mean year of birth of 1963 (range 1942-1969) and a percentage of male subjects of 44, in cohort study AE, 198 subjects with a mean year of birth of 1913 (1892-1927) and 47\% male subjects, and in cohort study RE, 298 subjects with a mean year of birth of 1906 (1889-1927) and 27\% male subjects. Table 2 also presents the numbers of selected subjects per trial, according to the number of previous vaccinations.

\section{Pre- and post-vaccination titres}

Figure 1 presents pre- and post-vaccination GMTvalues and proportions of protected subjects according to influenza (sub)types, trials and status of previous vaccination. Per trial, pre-GMT and pre-PR were, in 
Sero-response to repeated influenza vaccination: W.E.P. Beyer et al.

Table 2 Numbers of vaccinees, and exclusions from serological analysis

\begin{tabular}{|c|c|c|c|c|c|c|c|c|c|c|c|}
\hline \multirow[b]{2}{*}{ Trial $^{a}$} & & \multirow{2}{*}{$\begin{array}{l}\text { Total } \\
\text { number of } \\
\text { vaccinees } \\
\text { per trial }\end{array}$} & \multirow[b]{2}{*}{$\begin{array}{l}\text { Excluded } \\
\text { for age }\end{array}$} & \multirow{2}{*}{$\begin{array}{l}\text { Excluded for } \\
\text { previous } \\
\text { vaccinations }\end{array}$} & \multirow{2}{*}{$\begin{array}{l}\text { No } \\
\text { effect } \\
\text { group }\end{array}$} & \multirow{2}{*}{$\begin{array}{l}\text { No } \\
\text { complete } \\
\text { serology }\end{array}$} & \multirow[b]{2}{*}{$\begin{array}{l}\text { Selected } \\
\text { vaccinees }\end{array}$} & \multicolumn{4}{|c|}{ Number of previous vaccinations } \\
\hline & & & & & & & & 0 & 1 & 2 & 3 \\
\hline \multirow[t]{3}{*}{ YA } & 1986 & 143 & & 3 & 140 & & & & & & \\
\hline & 1987 & 130 & & 5 & & 7 & 118 & 25 & 93 & & \\
\hline & 1988 & 149 & & 4 & & 2 & 143 & 63 & 18 & 62 & \\
\hline \multirow[t]{4}{*}{$A E$} & 1986 & 63 & & 10 & 53 & & & & & & \\
\hline & 1987 & 116 & & 10 & & 3 & 103 & 40 & 63 & & \\
\hline & 1988 & 139 & & 10 & & 3 & 126 & 54 & 28 & 44 & \\
\hline & 1989 & 113 & & 6 & & 6 & 101 & 41 & 0 & 22 & 38 \\
\hline \multirow[t]{4}{*}{ RE } & 1986 & 234 & 7 & 4 & 223 & & & & & & \\
\hline & 1987 & 209 & 6 & 4 & & 5 & 194 & 59 & 135 & & \\
\hline & 1988 & 196 & 5 & 7 & & 25 & 159 & 40 & 25 & 94 & \\
\hline & 1989 & 223 & 10 & 16 & & 22 & 175 & 64 & 18 & 19 & 74 \\
\hline $\begin{array}{l}\text { All trials } \\
\text { No. subjects }\end{array}$ & & $\begin{array}{l}1715 \\
884\end{array}$ & $\begin{array}{l}28 \\
13\end{array}$ & $\begin{array}{l}79 \\
21\end{array}$ & $\begin{array}{l}416 \\
127\end{array}$ & $\begin{array}{l}73 \\
42\end{array}$ & $\begin{array}{l}1119 \\
681\end{array}$ & 386 & 380 & 241 & 112 \\
\hline
\end{tabular}

aYA, young adults; AE, ambulatory elderly; RE, resident elderly. ${ }^{D}$ Numbers of subjects are smaller than numbers of vaccination events (all trials) as a part of the subjects was vaccinated more than once

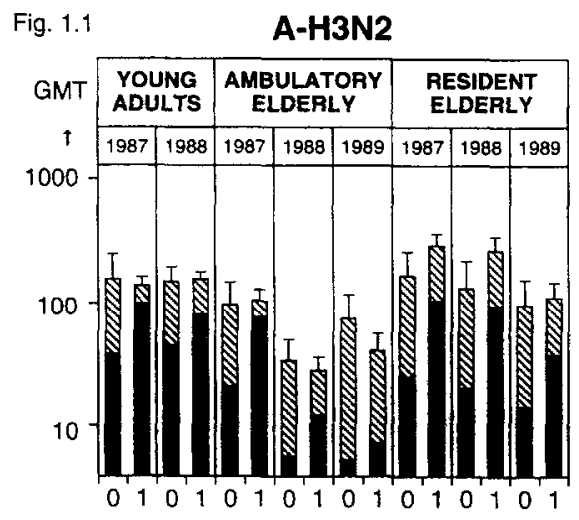

Fig. 1.2

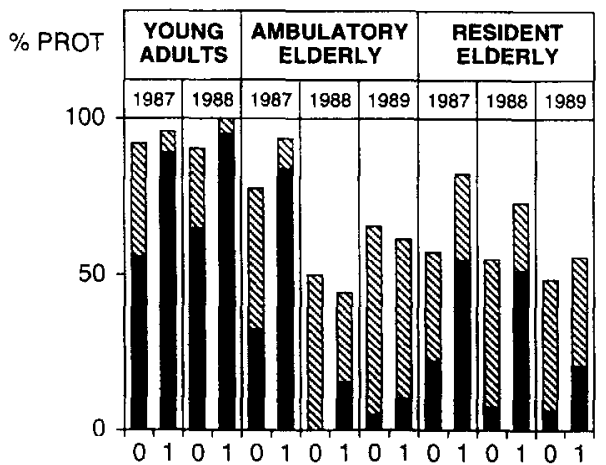

A-H1N1

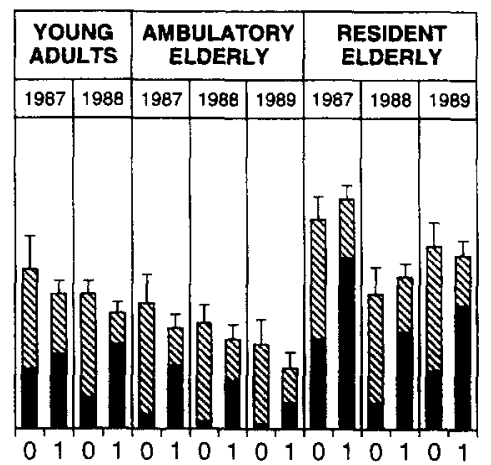

A-H1N1

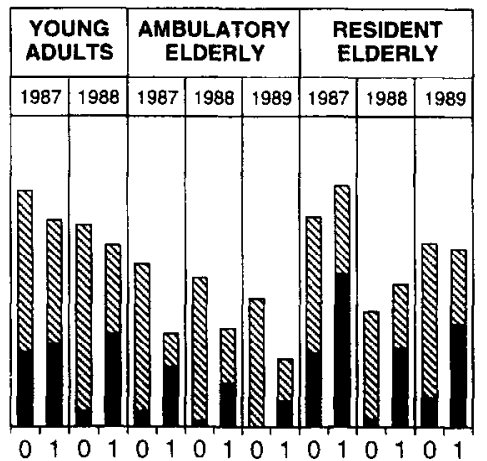

B

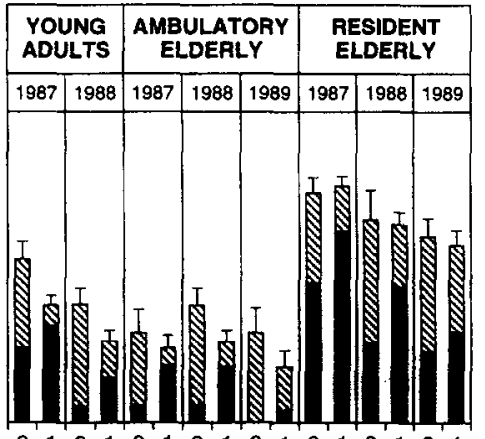

010101010101010101

PREVAC

$\mathbf{B}$

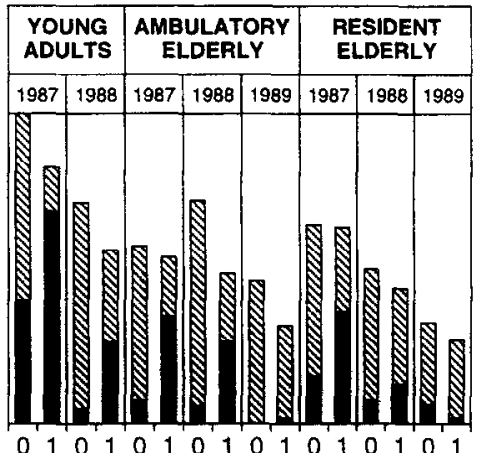

Figure 1 Pre- and post-vaccination mean titres, and proportions of protected subjects, according to trial and status of previous vaccination. (1.1) shows the geometric mean titres (GMT), (1.2) the proportion of protected subjects (\% PROT). The whole bar represents the post-vaccination titre (1.1), or the post-vaccination proportion of protected subjects (1.2); the lower part of the bar represents the pre-vaccination titre, or the pre-vaccination proportion of protected subjects. T, upper $95 \%$ confidence limit of post-vaccination titre (1.1); PREVAC, status of previous vaccination ( 0 , control group-not previously vaccinated; 1 , effect group-previously vaccinated)

virtually all cases, higher in previously vaccinated groups than in the control groups, regardless of the age-class and the antigenic resemblance of the consecutive virus strains per (sub)type. Post-GMT and post-PR were much less uniform: In previously vaccinated groups, in some cases these variables were higher, in other cases lower than the respective control groups. For the influenza A strains, no large differences could be detected in most cases. For influenza B, however, there appeared a general tendency to lower post-GMT and post-PR in groups with previous vaccination.

\section{Effect measures}

Figure 2 showing three different effect measures according to trial and (sub)type, reveals that the generally positive effect of previous vaccination on prevaccination titres was significant in the majority of trials. Only one trial (RE 1989 for influenza B) had an insignificantly lower percentage of protected subjects previously vaccinated compared to the control group. In contrast, a clear-cut effect of previous vaccination on the post-vaccination status was not present: most values, either positive or negative, were not significantly 
Pre-vaccination titre
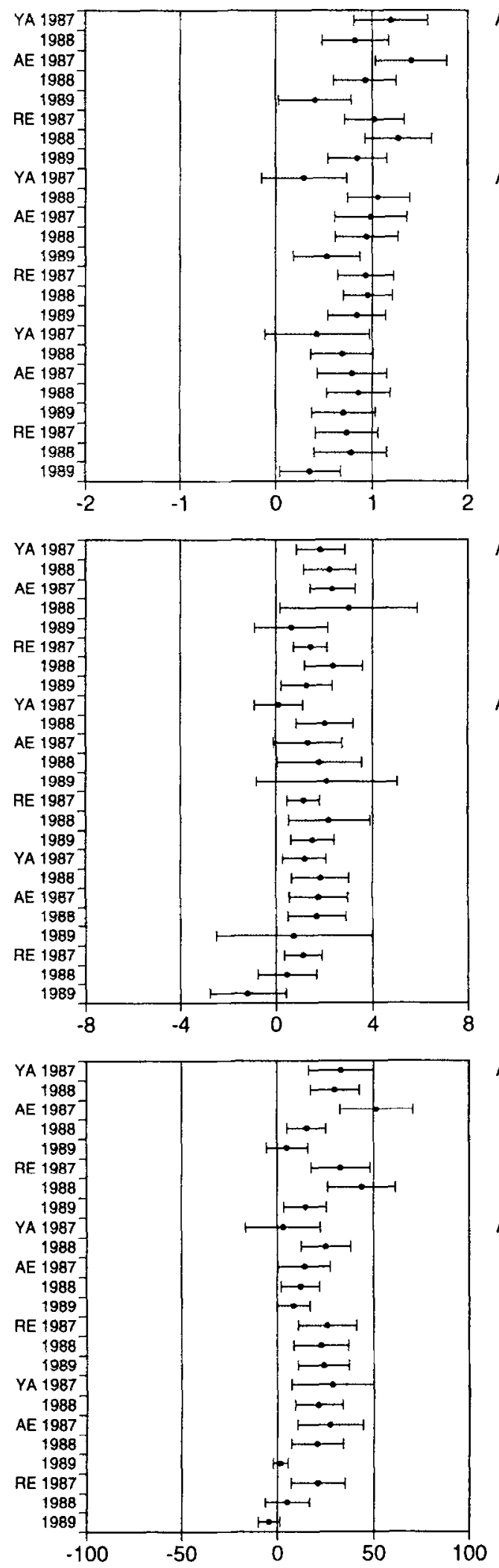

\section{Post-vaccination titre}
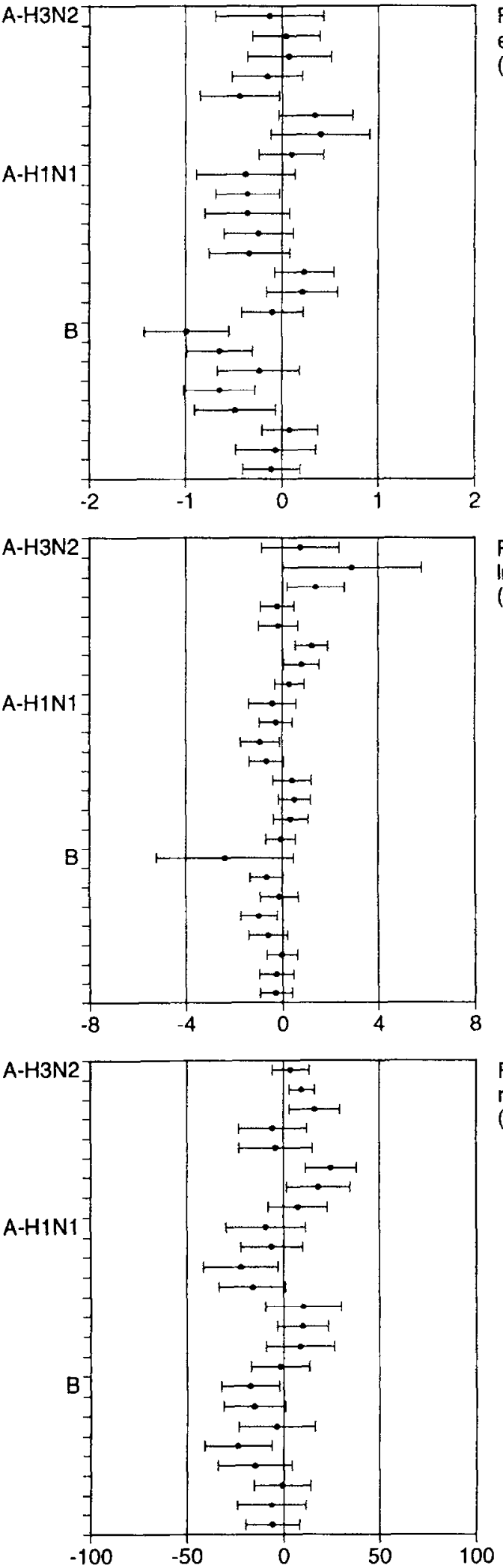

Fig. 2.1

effect size

(ES)

Fig. 2.2

In Odds Ratio

(InOR)

Fig. 2.3

rate difference

$(\mathrm{RD})$

Figure 2 Effect measures (with 95\% Cls) according to trial and (sub)type. YA, young adults; AE, ambulatory elderly; RE, resident elderly

different from zero. $E S$-values varied between -0.99 and +0.51 , In $O . R$. -values between -2.38 and +2.90 , and $R D$-values between $-23.6 \%$ and $+24.6 \%$. It appeared, however, that there were differences between (sub)types: A-H3N2-values were generally higher than B-values.
This impression was confirmed, for both pre- and post-vaccination titres, by pooling the effect measures of all eight trials according to (sub)types (Figure 3). In all 18 comparisons, influenza A-H3N2 had higher values than influenza $\mathrm{B}$, with $\mathrm{A}-\mathrm{H} 1 \mathrm{~N} 1$ in an intermediate position. For pre-vaccination titres, this sequence did 

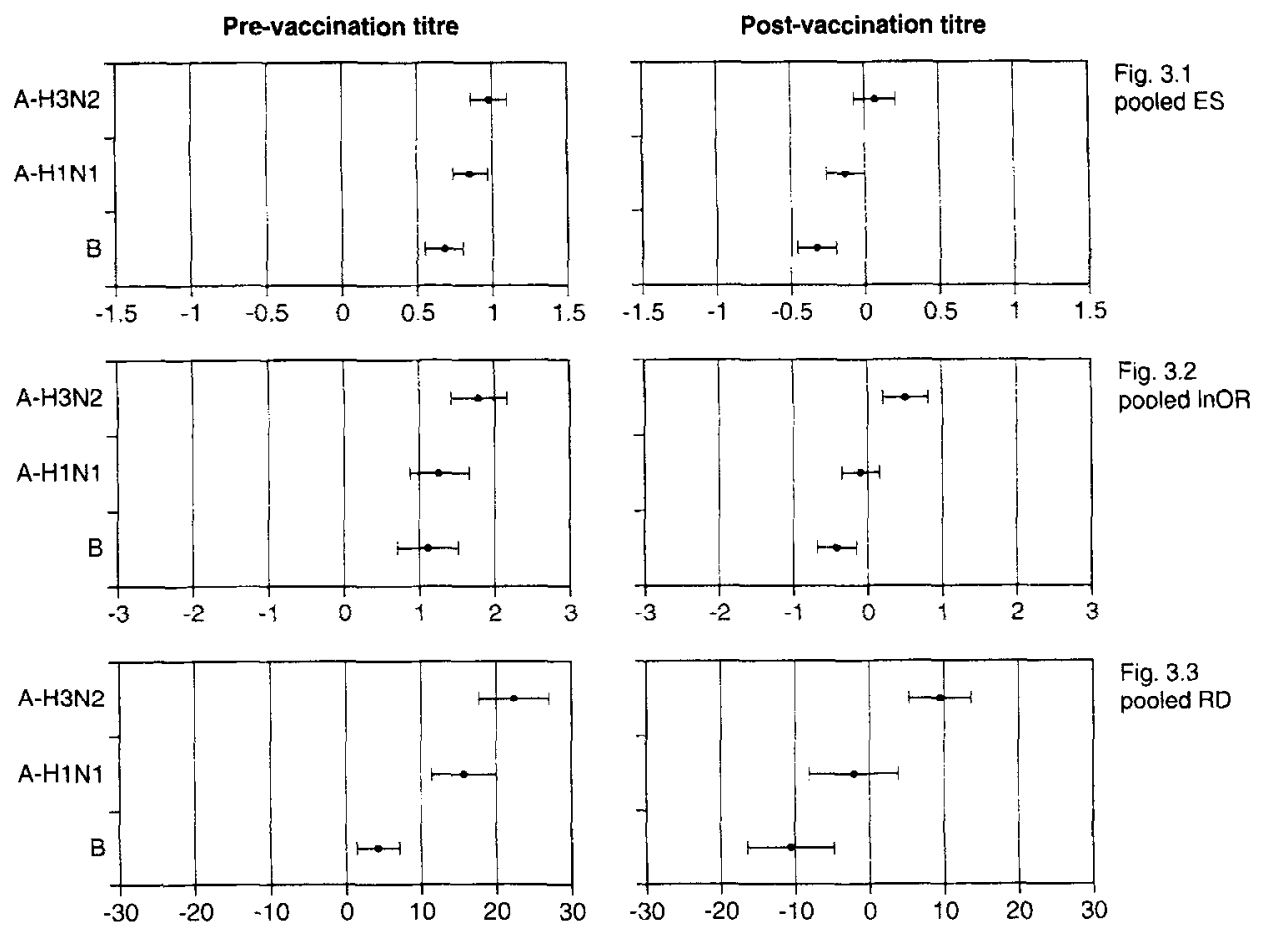

Fig. 3.3

pooled RD

Figure 3 Pooled effect measures (with $95 \% \mathrm{Cls}$ ), according to (sub)type. ES, effect size; In O.R., logarithm of odds ratio; RD, rate difference

not lead to a qualitative difference between (sub)types: all pooled effect measures were significantly positive. The average additional effect of previous vaccination on pre-vaccination proportion of protected subjects (Figure $3 c$ ) was $+22.3 \%$ for $\mathrm{A}-\mathrm{H} 3 \mathrm{~N} 2,+15.8 \%$ for $\mathrm{A}-\mathrm{H} 1 \mathrm{~N} 1$ and $+4.2 \%$ for $B$. In contrast, the pooled effect measures of post-vaccination titres were positive only for A-H3N2 (pooled $\ln O . R$, and $R D$ even significantly so), negative but very close to zero for A-H1N1, and significantly negative for $B$. In other words, on average previous vaccination is associated with higher post-vaccination titres for A-H3N2 vaccine components, compared with not previously vaccinated subjects, and with lower titres for $B$ components. There is no such effect for the A-H1N1 component. The average increase of the postvaccination proportion of protected subjects, due to previous vaccination, was $+9.4 \%(95 \% \mathrm{CI}:+5.3$ to $+13.6 \%)$, the average decrease for $\mathrm{B}$ was $-10.6 \%$ $(-16.5$ to $-4.8 \%)$, and for A-H1N1 $-2.1 \%(-8.1 \%$ to $+3.9 \%$ ) (Figure $3 c$ ).

\section{Variables influencing post-vaccination titres}

Per trial and (sub)type, and in part within relevant subpopulations, post-vaccination titres and postvaccination protection were subjected to linear regression and logistic regression, respectively, including a number of independent variables. Year of birth within the given range of the cohort study $(\geq 1928$ for YA, $<1928$ for AE and RE), gender, days between vaccination and drawing of post-vaccination blood specimen, number of previous vaccinations, underlying diseases (for cohort studies $\mathrm{AE}$ and $\mathrm{RE}$ ), vaccine dosages in 1986 (for cohort study YA and RE), and clinically or virologically confirmed illness in January/February 1986 and March 1989 (for cohort study RE) did not show a significant contribution to post-vaccination titres. In all comparisons, the pre-vaccination titre was the strongest predictor of the post-vaccination status. In most com- parisons, also status of previous vaccination contributed significantly to post-vaccination titres.

Linear regression on post-vaccination titres was repeated with only pre-vaccination titres and status of previous vaccination as independent factors. The resulting regression coefficients for both factors are shown in Table 3. All regression coefficients for pre-vaccination titre were significantly 0 , confirming the well-known strong positive linear relationship between pre- and post-vaccination titres. Regression coefficients for the status of previous vaccination, all but one, were negative. The addition of an interaction term (the product of pre-vaccination titre and status of previous vaccinations) did not improve the model.

\section{DISCUSSION}

The first main result of our paper concerns the prevaccination titres: they are generally higher in effect groups (previously vaccinated) than in control groups (not previously vaccinated). This finding is biologically plausible (persistence of vaccine-induced antibody) and has been previously described in other papers $5,7-12,27$.

Our second main finding, on post-vaccination titres, is not clear-cut: post-vaccination titres of effect groups can be higher than, equal to, or lower than those in control groups. When pooling the effect measures of all trials according to (sub)type, we saw a tendency to higher post-vaccination titres for the A-H3N2 vaccine components, the opposite for B components and virtually no effect for A-H1N1. This also implies that the sequence of consecutive vaccine-strains in the period 1986-1989 had obviously no influence. The (sub)type difference of effect measures is not reflected by the fact that both A-H3N2 and $\mathbf{B}$ vaccine components changed more frequently than the A-H1N1 component.

In this paper, we examined three different study populations. Age differences could have affected the 
Sero-response to repeated influenza vaccination: W.E.P. Beyer et al.

Table 3 Linear regression of pre-vaccination titre and status of previous vaccinations on post-vaccination titre per (sub)type and trial

\begin{tabular}{|c|c|c|c|c|c|c|}
\hline \multirow[b]{2}{*}{ (Sub)type } & \multirow[b]{2}{*}{ Trial $^{a}$} & & \multicolumn{2}{|l|}{ Pre-vaccination titre } & \multicolumn{2}{|c|}{ Status of previous vaccination ${ }^{b}$} \\
\hline & & & Regression coefficient & $P$-value & Regression coefficient & $P$-value \\
\hline \multirow[t]{8}{*}{ H3N2 } & YA & 1987 & 0.707 & $<0.0001$ & -0.339 & $<0.0001$ \\
\hline & & 1988 & 0.787 & $<0.0001$ & -0.180 & 0.0010 \\
\hline & $A E$ & 1987 & 0.783 & $<0.0001$ & -0.406 & $<0.0001$ \\
\hline & & 1988 & 1.037 & $<0.0001$ & -0.434 & $<0.0001$ \\
\hline & & 1989 & 0.744 & $<0.0001$ & -0.360 & 0.0010 \\
\hline & RE & 1987 & 0.352 & $<0.0001$ & 0.019 & 0.8429 \\
\hline & & 1988 & 0.703 & $<0.0001$ & -0.161 & 0.0987 \\
\hline & & 1989 & 0.625 & $<0.0001$ & -0.199 & 0.0571 \\
\hline \multirow{8}{*}{ H1N1 } & YA & 1987 & 0.595 & $<0.0001$ & -0.274 & 0.0111 \\
\hline & & 1988 & 0.635 & $<0.0001$ & -0.433 & $<0.0001$ \\
\hline & $\mathrm{AE}$ & 1987 & 0.662 & $<0.0001$ & -0.464 & $<0.0001$ \\
\hline & & 1988 & 0.869 & $<0.0001$ & -0.409 & $<0.0001$ \\
\hline & & 1989 & 0.990 & $<0.0001$ & -0.354 & 0.0005 \\
\hline & $\mathrm{RE}$ & 1987 & 0.538 & $<0.0001$ & -0.199 & 0.0334 \\
\hline & & 1988 & 0.634 & $<0.0001$ & -0.230 & 0.0320 \\
\hline & & 1989 & 0.467 & $<0.0001$ & -0.317 & 0.0020 \\
\hline \multirow[t]{8}{*}{$\mathrm{B}$} & YA & 1987 & 0.646 & $<0.0001$ & -0.488 & $<0.0001$ \\
\hline & & 1988 & 0.713 & $<0.0001$ & -0.464 & $<0.0001$ \\
\hline & $\mathrm{AE}$ & 1987 & 0.732 & $<0.0001$ & -0.351 & 0.0002 \\
\hline & & 1988 & 0.714 & $<0.0001$ & -0.518 & $<0.0001$ \\
\hline & & 1989 & 1.503 & $<0.0001$ & -0.411 & 0.0004 \\
\hline & RE & 1987 & 0.519 & $<0.0001$ & -0.178 & 0.0058 \\
\hline & & 1988 & 0.484 & $<0.0001$ & -0.262 & 0.0099 \\
\hline & & 1989 & 0.545 & $<0.0001$ & -0.166 & 0.0656 \\
\hline
\end{tabular}

a YA, young adults; AE, ambulatory elderly; RE, resident elderly. ${ }^{b}$ Status of previous vaccination: 0 for not previously vaccinated; 1 for previously vaccinated

effect measures, both by mechanisms of biological ageing of the immune system (decrease of seroresponsiveness, for review see Refs 23,24), and the influenza-specific phenomenon of "original antigenic $\sin ^{\prime 25}$. Figure 2 reveals, however, that there are no real differences between the effect measures of the cohort studies YA (young adults, most of whom born after 1957) and AE (ambulatory elderly, born in 1927 or earlier). In particular this was true for influenza A-H1N1, the subtype of which the seroresponse is usually strongest affected by the original antigenic sin as it had disappeared in 1957 and reappeared in $1977^{26}$

Other differences between our study populations concern the influenza vaccine types (subunit vaccine in YA and AE, whole-virus vaccine in RE), and the epidemiological characteristics: RE consisted of a semiclosed community (nursing home), while the vaccinees of $\mathrm{AE}$ and YA belonged to the general, epidemiologically open, population. Natural influenza, like the A-H3N2 epidemic in RE in 1986, has a much stronger impact in semi-closed communities which may modulate the response on vaccination in the following years. However, we did not find, by regression analysis, any such influence in our data. Another difference may be that the groups of previously not-vaccinated subjects were better defined in $\mathrm{AE}$ and YA than in RE where subjects with previous vaccination outside the nursing home could not always be identified because of the high proportion of dementia. We believe that the combined influence of all these population characteristics did not seriously affect our outcome measures, as can be derived from the only small inter-trial variation (Figure 2).

Serum titrations were done per trial, on different points of time, in two different laboratories, and with different protocols of the HI test. This approach did not allow for comparisons between trials, and specially not for the follow-up of those individuals who had participated during the whole study period. Such an individual follow-up would have been statistically stronger, but only possible for the A-H1N1 strain A/Taiwan/1/86 which remained in the vaccine composition during the whole study period, while the A-H3N2 and B components were replaced by new strains every 1 or 2 years. Our approach, a comparison of effect and control groups strictly per trial, allowed to ignore the between-trial differences of A-H3N2 and B components, and the differences due to titration methods. A possible statistical inferiority, compared with individual followup, may have been compensated by the large number of data (1119 paired sera).

To compare our finding on post-vaccination titres with the international literature, we identified nine papers wherein rate differences could be calculated. Table 4 shows the results of, in total, 34 comparisons. In 26 comparisons, rate differences were not significantly different from zero (i.e. no effect of previous vaccination). In two comparisons, rate differences were significantly higher in the effect groups (one time $\mathrm{A}-\mathrm{H} 3 \mathrm{~N} 2$, one time $\mathrm{A}-\mathrm{H} 1 \mathrm{~N} 1$ ), and in six comparisons rate differences were significantly lower (one time A-H3N2, two times A-H1N1, three times B). Of special interest were the studies of Govaert et $a .^{7}$ and Glathe et $a l^{9}$. Both studies were performed in the same year (i.e. identical strains) with the same vaccine type (split-virus). Nevertheless, Govaert et al. saw a strong negative effect for all three (sub)types, and Glathe et al. a strong positive effect for the A-strains. Although the results of Govaert et al. may be more reliable than those of Glathe et al. because of larger numbers of vaccinees (906 vs 92) and a more consistent statistical analysis, this extreme heterogeneity is remarkable.

We saw in our data that an individual postvaccination titre is strongly dependent on the prevaccination titre and, inversely, on the status of previous vaccination. The linear relationship between pre- and post-vaccination titre is well known ${ }^{29}$. The inverse 
Sero-response to repeated influenza vaccination: W.E.P. Beyer et al.

Table 4 Review of the literature: effect of previous vaccinations on post-vaccination percentage of protected subjects

Post-vaccination rate difference $(95 \% \mathrm{Cl})$ per (sub)type $(\%)^{\circ}$

\begin{tabular}{|c|c|c|c|c|c|}
\hline First author & Year of trial & Number of vaccinees & $\mathrm{A}-\mathrm{H} 3 \mathrm{~N} 2$ & $\mathrm{~A}-\mathrm{H} 1 \mathrm{~N} 1$ & $B$ \\
\hline $\begin{array}{l}\text { Powers }^{b} \\
\text { Powers }^{b} \\
\text { Keitel }^{c} \\
\text { Keitel }^{c} \\
\text { Peters }^{d} \\
\text { Gross }^{e} \\
\text { Beyer }^{f} \\
\text { McElhaney }^{g} \\
\text { Govaert }^{h} \\
\text { Glathe } \\
\text { Pyhälä } \\
\text { Pyhälä } \\
\text { This paper }\end{array}$ & $\begin{array}{l}1981 \\
1981 / 82 \\
1983 \\
1984 \\
1985 \\
1986 \\
1987 \\
1991 \\
1991 \\
1991 \\
1991 \\
1992 \\
\text { Pooled data }\end{array}$ & $\begin{array}{l}52 \\
70 \\
309 / 316 \\
457 / 461 \\
131 \\
140 \\
108 \\
26 \\
906 \\
92 \\
33 \\
45\end{array}$ & $\begin{array}{l}-11.5(-24.2-1.1) \\
2.9(-2.7-8.4) \\
1.6(-12.8-9.5) \\
6.9(-16.3-2.5) \\
0.6(-19.1-20.3) \\
8.7(-28.1-10.8) \\
\pm 0 \\
-18.4(-27.4--9.3) \\
14.7(5.1-24.3) \\
-6.9(-41.1-27.2) \\
1.5(-22.4-25.5) \\
9.4(5.3-13.6)\end{array}$ & $\begin{array}{l}11.5(-7.0-30.1) \\
8.6(-5.5-22.6) \\
-0.9(-8.2-6.3) \\
-9.9(-19.3--0.6) \\
-10.0(-30.7-10.7) \\
-5.5(-23.4-12.5) \\
\pm 0 \\
-26.6(-36.2--17.0) \\
17.1(4.6-29.7) \\
-1.4(-26.4-23.6) \\
4.5(-17.9-27.0) \\
-2.1(-8.1-3.9)\end{array}$ & $\begin{array}{l}7.7(-9.7-25.1) \\
0.0(-7.8-7.8) \\
-8.0(-16.3-0.3) \\
-3.1(-6.5-0.2) \\
<0 \\
-1.5(-20.2-17.2) \\
-20.5(-38.7--2.2) \\
\pm 0 \\
-32.4(-42.0--22.7) \\
5.4(-4.2-15.0) \\
1.4(-29.9-32.7) \\
-7.6(-35.0-19.9) \\
-10.6(-16.4--4.8)\end{array}$ \\
\hline
\end{tabular}

${ }^{a}$ Rate difference $(R D)$, difference between the proportions of protected subjects of effect group (previously vaccinated) and control group (not previously vaccinated). Significant $R D$-values bold. ${ }^{b}$ Powers et al ${ }^{10}$, in a time-cohort study, immunized $18-65$-year-old healthy adults with whole-virus vaccine containing A/Bangkok/1/79 (H3N2), A/Brazil/11/78 (H1N1) and B/Singapore/222/79 (7.5 or $15 \mu \mathrm{g}$ HA per strain) three times with time-intervals of 6 months. Protective titre threshold: 40 . Data estimated from Fig. 1 and Fig. 2 of the paper, with "1st vaccination" as control group, and "2nd vaccination" as effect group. Data on the third vaccination not included. ${ }^{\circ}$ Keitel et al. ${ }^{5}$ immunized 30-60-year-old adults with whole-virus vaccine containing A/Philippines/2/82 (H3N2), A/Brazil/11/78 (H1N1) and B/Singapore/222/79, or placebo, in 1983, and with A/Philippines/2/82 (H3N2), A/Chile/1/83 (H1N1), and B/USSR/100/83, or placebo, in 1984 (15 $\mu \mathrm{g} \mathrm{HA}$ per strain). Protective titre threshold: 32 . Data derived from Tables 1 and 2 of the paper. Data on placebo not included. "Peters et al. ${ }^{8}$ described a dose response trial in elderly subjects with a whole-virus vaccine containing A/Philippines/2/82 (H3N2), A/Chile/1/83 (H1N1), and B/USSR/100/83 (15 $\mu$ g HA per strain), or B/USSR/100/83 (45 $\mu \mathrm{g} \mathrm{HA})$ alone. They did not present percentages of protection for groups previously and not previously vaccinated, but showed that post-vaccination GMT for the influenza B vaccine component was significantly lower in previously vaccinated subjects, compared to those not previously vaccinated. This finding was indicated by the symbol " $<0$ " in this table. "Gross et al. ${ }^{11}$ immunized $65-96$-year-old healthy ambulatory and institutionalized elderly adults with split-virus vaccine containing $A / M i s s i s s i p p i / 1 / 85$ (H3N2), A/Chile/1/83 (H1N1) and B/Ann Arbor/1/86 (15 $\mu \mathrm{g} \mathrm{HA}$ per strain). Protective titre threshold: 40 . Data derived from Table 3 of the paper. Data on a smaller group receiving also A/Taiwan/1/86 (H1N1) 1 month later, not included. 'Beyer et al. ${ }^{27}$ vaccinated patients on chronic intermittent haemodialysis with whole-virus vaccine containing A/Leningrad/360/86 (H3N2), ATTaiwan/1/86 (H1N1) and B/Ann Arbor/1/86 (10, 10, and $15 \mu \mathrm{g} \mathrm{HA})$. Protective titre threshold: 100 for influenza A, 200 for influenza B. The publication presented data on the effect of previous vaccinations as "mean fold increase". Orlginal raw data were re-analysed for this paper. ${ }^{9} \mathrm{McEl}$ (haney et al. ${ }^{12}$ studied whole- and split-virus vaccines containing $\mathrm{A} / \mathrm{Beijing} / 353 / 89$ (H3N2), A/Taiwan/1/86 ( $\mathrm{H} 1 \mathrm{~N} 1)$ and B/Panama/45/90, in young and elderly adults; they did not present data on previous vaccinations but stated that "subgroup analysis showed that previous vaccination had no effect on the response to the different strains measured in the study" ( $p$. 1059). This finding was indicated by the symbol " \pm 0 " (i.e. not significantly different from 0 ) in this table. "Govaert et al. ${ }^{7}$ immunized $60-91$-year-old healthy ambulatory elderly adults with split-virus vaccine containing A/Beijing/353/89 (H3N2), A/Taiwan/1/86 (H1N1), B/Panama/45/90 and B/Beijing/1/87 (15 $\mu \mathrm{g}$ HA per strain). Protective titre threshold: 100 for influenza A, 200 for influenza B. Data derived from Table 10.3 of the paper. Data on the second B-strain not included. Glathe et al. ${ }^{9}$ compared the seroresponse to a split-virus vaccine containing A/Beijing/353/89 (H3N2), A Taiwan/1/86 (H1N1) and B/Yamagata/16/88 (15 $\mu \mathrm{g} \mathrm{HA}$ per strain), in five groups of young and elderly adults with different proportions of previous vaccinations. Protective titre threshold: 40 . Authors did not perform a statistical analysis on the effect of previous vaccination. Data derived from Tables 2-4 of the paper, with "Group A" (not previously vaccinated subjects) as control group, and "Group E" (95\% previously vaccinated) as effect group. Data on groups $B$ to $D\left(26 \%-45 \%\right.$ previously vaccinated) not included. 'Pyhälä et al. ${ }^{28}$ followed three groups of adult volunteers (25-59 years of age) during 1990-1992. The split-virus vaccine contained, in 1991, A/Beijing/353/89 (H3N2), A/Taiwan/1/86 (H1N1) and B/Panama/45/90, and in 1992, A/Beijing/353/89 (H3N2), A/Taiwan/1/86 (H1N1) and B/Yamagata/16/88 (15 $\mu$ g HA per strain). Protective titre threshold: 40. Data were derived from Table 2 of the paper, with "group 1" as effect group and "group 2" as control group in 1991, and "group 1" and "group 2" as pooled effect group and "group 3" as control group in 1992. Data for A/Taiwan/1/86 (H1N1) and B/Panama/45/90 were not given. Calculations are based on data for A/Finland/164/91 (H1N1) and B/Yamagata/16/88

relationship between status of previous vaccination and post-vaccination titre has already been seen by Peters et $a l^{8}$ and Pyhälä et al. ${ }^{28}$ At a given pre-vaccination titre, the post-vaccination titre can be expected to be lower in previously vaccinated subjects than in not previously vaccinated subjects. Thus, natural antibody, caused by previous infections, has a larger potential to form high post-vaccination titres, than the same amount of vaccine-induced antibody ${ }^{30}$.

The biological interpretation of the inverse relationship between status of previous vaccination and postvaccination titre is troublesome. The phenomenon could be described as a "negative booster effect of previous vaccinations" suggesting an active immunological feed-back mechanism which inhibits the production of additional post-vaccination antibody. It has also been pointed out that natural and vaccine-induced antibody may differ in their IgA- and IgG-subclass composition or their ratio of strain-specific and crossreacting components which are not differentiated by the HI-test. It is also possible that the inverse relationship is a pure "mathematical artifact" in the sense that postvaccination antibody may reach a plateau after first vaccination which can not be exceeded by repeated vaccinations. Since at the same time pre-vaccination titre is higher after repeated vaccination, a negative coefficient for repeated vaccinations occurs in the regression equation which has no biological meaning at all.

It should be underlined that our data were based on the HI-titre, i.e. a surrogate marker for real vaccine efficacy. In high concentrations, the HI-titre is a good marker, or in other words: a vaccine which induces high HI-titres, is undoubtedly a highly effective means to prevent serious influenza illness. On the other side, a vaccine, which induces low III-titre should not automatically be regarded as inferior if it stimulates other immunological measures which are also protective even without high HI-titres. Therefore, our finding that, specially in cohort studies AE and YA, post-vaccination HI-antibody decreased after annually repeated vaccination for the influenza B component, does not necessarily 
mean that real protection against natural influenza $B$ evenly decreased in these groups. In studies which recorded natural infections in groups with and without repeated vaccinations, subjects with previous vaccination showed an equal or even greater protection against natural influenza compared to those without ${ }^{5-7}$.

Thus, while annually repeated influenza vaccination has probably no negative clinical impact, it should always be included as an independent factor in serological vaccination studies.

\section{ACKNOWLEDGEMENTS}

The study was financially supported by The Foundation of Respiratory Virology, notably Influenza (SRVI), Rotterdam, The Netherlands. The authors wish to thank all volunteers for their cooperation, Professor A.S. Monto for stimulating discussion, Dr R.J.A. Diepersloot and Dr E. Remarque for their valuable comment on the manuscript, and Ms C. Kruyssen for help in preparing the manuscript.

\section{REFERENCES}

1 Hoskins, T.W., Davis, J.R., Allchin, A., Miller, C.L. and Pollock, T.M. Controlled trial of inactivated influenza vaccine containing the A/Hong Kong strain during an outbreak of influenza due to the A/England/42/72 strain. Lancet 1973, ii, 116-120

2 Hoskins, T.W., Davis, J.R., Smith, A.J., Allchin, A., Miller, C.L. and Pollock, T.M. Influenza at Christ's Hospital: March 1974. Lancet 1976, i, 105-108

3 Hoskins, T.W., Davis, J.R., Smith, A.J., Miller, C.L and Allchin, A. Assessment of inactivated influenza $A$ vaccine after three outbreaks of influenza at Christ's Hospital. Lancet 1979, i, 33-35

4 Nicholson, K.G. Annual vaccination: conclusions and recommendations. In: Options for the Control of Influenza II. Proceedings of the International Conference on Options for the Control of Influenza, Courchevel, 27 September-2 October 1992 (Eds Hannoun, C., Kendal, A.P., Klenk, H.D. and Ruben, F.L.). Elsevier Science, Amsterdam, 1993, pp. 451-455

5 Keitel, W.A., Cate, T.R. and Couch, R.B. Efficacy of sequential annual vaccination with inactivated influenza virus vaccine. Am J. Epidemiol. 1988, 127, 353-364

6 Gross, P.A. Denning, C.R., Gaerlan, P.F. et al. Benefit of annual influenza immunization in patients with cystic fibrosis. In: Options for the Control of Influenza 11. Proceedings of the International Conference on Options for the Control of Influenza, Courchevel, 27 September-2 October 1992 (Eds Hannoun, C., Kendal, A.P., Klenk, H.D. and Ruben, F.L.). Elsevier Science, Amsterdam, 1993, pp. 417-421

7 Govaert, T.M.E., Sprenger, M.J.W., Dinant, G.J., Aretz, K., Masurel, N. and Knottnerus, J.A. Immune response to influenza vaccination of elderly people. A randomized double-blind placebo-controlled trial. Vaccine 1994, 12, 1185-1189

8 Peters, N.L., Meiklejohn, G. and Jahnigen, D.W. Antibody response of an elderly population to a supplemental dose of influenza B vaccine. J. Am. Geriatrics Soc. 1988, 36, 593599

9 Glathe, H., Bigl, S. and Grosche, A. Comparison of humoral immune responses to trivalent influenza split vaccine in young, middle-aged and elderly people. Vaccine 1993, 11, 702705

10 Powers, R.D., Hayden, F.G., Samuelson, J. and Gwaltney, J.M Immune response of adults to sequential influenza vaccination. J. Med. Virol. 1984, 14, 169-175
11 Gross, P.A., Quinnan, G.V., Weksler, M.E., Setia, U. and Douglas, R.G. Relation of chronic disease and immune response to influenza vaccine in the elderly. Vaccine 1989, 7, 303-308

12 McElhaney, J.E., Meneilly, G.S., Lechelt, K.E., Beattie, B.L. and Bleackley, R.C. Antibody response to whole-virus and split-virus influenza vaccines in successful ageing. Vaccine 1993, 10, $1055-1060$

13 Palache, A.M. Influenza subunit vaccine-ten years experience. Eur. J. Clin. Res. 1992, 3, 117-138

14 Hendriksen, E., Beyer, W.E.P., Kluver, H.F., Den Ronden-De Jong, J. and Masurel, N. Influenza and influenzaprofylaxis in a nursing home (Dutch). Vox Hospitii 1988, 12, 81-86

15 Dowdle, W.R., Kendal, A.P. and Noble, G.R. Influenza viruses. In: Diagnostic Procedures for Viral, Rickettsialand Chlamydial Infections, 5th edn (Eds Lenette, E.H. and Schmidt, N.J.) American Public Health Association, Washington, 1979, pp. 585-609

16 Masurel, N., Ophof, P. and De Jong, P. Antibody response to immunization with influenza A/USSR/77 (H1N1) virus in young individuals primed or unprimed for A/New Jersey/76 (H1N1) virus. J. Hyg. (Camb) 1981, 87, 201-209

17 Berlin, B., McQueen, J., Minuse, E. and Davenport, F. A method for increasing the sensitivity of the hemagglutinationinhibition test with equine influenza virus. Virology 1963, 21, 665-666

18 Wesselius-De Casparis, A., Masurel, N. and Kerrebijn, K.F. Field trial with human and equine influenza vaccines in children: protection and antibody titres. Bull. WHO 1972, 46, 151-157

19 Beyer, W.E.P., Teunissen, M.W.E., Diepersloot, R.J.A. and Masurel, N. Immunogenicity and reactogenicity of two doses of a trivalent influenza split vaccine. An open randomized study in healthy, unprotected, adult volunteers. J. Drugther. Res. 1986, 9, 369-374

20 Dawson-Saunders, B. and Trapp, R.G. Basic and Clinical Biostatistics. Appleton and Lange, East Norwalk, CT, 1990. pp. 222-224

21 Yusuf, S., Peto, R., Lewis, J., Collins, R. and Sleight, P. Beta blockade during and after myocardial infarction: an overview of the randomized trials. Progr. Cardiovasc. Dis. 1985, 27, 335-371

22 Breslow, N. and Day, N.E. The analysis of case-control-studies. Stat. Meth. Cancer Res. Lyon, France (IARC) 1980, 1, 142

23 Beyer, W.E.P., Palache, A.M., Baljet, M. and Masurel, N. Antibody induction by influenza vaccines in the elderly: a review of the literature. Vaccine 1989, 7, 385-394

24 Remarque, E.J., Van Beek, W.C.A., Ligthart, G.J. et al. Improvement of the immunoglobulin subclass response to influenza vaccine in elderly nursing-home residents by the use of high-dose vaccines. Vaccine 1993, 11, 649-653

25 Francis, T., Davenport, F.M. and Hennessy, A.V. A serologic recapitulization of human infection with different strains of influenza virus. Trans. Assoc. Am. Phys. 1953, 66, 231-239

26 Kendal, A.P. Nobel, G.R., Skehel, J.J. and Dowdle, W.R Antigenic similarity of influenza $A(\mathrm{H} 1 \mathrm{~N} 1)$ viruses from epidemics in 1977-1978 to "Scandinavian" strains isolated in epidemics of 1950-1951. Virology 1978, 89, 632-636

27 Beyer, W.E.P., Noordzij, T.C., Kramer, P. et al. Effect of immunomodulator thymopentin on impaired seroresponse to influenza vaccine in patients on haemodialysis. Nephron 1990, 54, 296-301

28 Pyhälä, R., Kumpulainen, V., Alanko, S. and Forsten, T. HI antibody kinetics in adult volunteers immunized repeatedly with inactivated trivalent influenza vaccine in 1990-1992. Vaccine 1994, 12, 947-952

29 Voth, D.W., Feldman, H.A. and Steinschneider, A. Comparative responses of elderly persons to aqueous and depot influenza vaccines. Arch. Environ. Hith. 1966, 13, 576-585

30 Davies, J.R. and Grilli, E.A. Natural and vaccine-induced antibody as a predictor of immunity in the face of natural challenge with influenza viruses. Epidem. Inf. 1989, 102, 325-333 